\title{
HPV testing for cervical cancer screening in Mozambique: challenges and recommendations
}

Cristina M. de Oliveira ${ }^{1}$ (0, Mila M. P. Salcedo ${ }^{2}$, Eliane Monteiro ${ }^{3}$, Arlete Mariano ${ }^{3}$, Mario J. C. Verdinho ${ }^{3}$, Reginaldo de Alice M Julião ${ }^{4}$, Kathryn Kundrod, Chelsey Smith ${ }^{5}$, Jennifer Carns ${ }^{5}$, Viviane Andrade ${ }^{6}$, Ellen Baker ${ }^{7}$, Natacha Phoolcharoen ${ }^{8}$, Rebecca R. Richards-Kortum ${ }^{5}$, José Humberto T. G. Fregnani ${ }^{9}$, Kathleen M. Schmeler ${ }^{7}$, Cesaltina Lorenzoni ${ }^{10}$

\begin{abstract}
${ }^{1}$ Molecular Oncology Research Center, Barretos Cancer Hospital, Barretos, Brazil; Institute of Life and Health Sciences (ICVS), University of Minho, Braga, Portugal, 2 The Department of Gynecologic Oncology \& Reproductive Medicine - The University of Texas MD Anderson Cancer Center, Houston, Texas, USA; The Obstetrics and Gynecology Department, Federal University of Health Sciences of Porto Alegre/Irmandade Santa Casa de Misericordia de Porto Alegre, Brazil, ${ }^{3}$ Hospital Geral e Centro de Saúde de Mavalane, Maputo, Mozambique, ${ }^{4}$ Ministerio da Saúde de Moçambique (MISAU), Maputo, Mozambique, 5 Rice University, Houston, Texas, USA, ${ }^{6}$ Research Support Center, Barretos Cancer Hospital, Barretos, Brazil, 7 The Department of Gynecologic Oncology \& Reproductive Medicine - The University of Texas MD Anderson Cancer Center, Houston, Texas, USA, 8 The Department of Gynecologic Oncology \& Reproductive Medicine - The University of Texas MD Anderson Cancer Center, Houston, Texas, USA; Department of Obstetrics and Gynecology, King Chulalongkorn Memorial Hospital, Bangkok, Thailand, 9 AC Camargo Cancer Center, São Paulo, Brazil, 10 Ministerio da Saúde de Moçambique (MISAU), Maputo, Mozambique; Universidade Eduardo Mondlane (UEM), Faculdade de Medicina, Maputo, Mozambique; Hospital Central de Maputo, Maputo, Mozambique

Keywords: hpv, cervical cancer, screening

https://doi.org/10.29392/001c.32432
\end{abstract}

\section{Journal of Global Health Reports}

Vol. 6, 2022

\section{Background}

Cervical cancer is the fourth most common cancer affecting women worldwide and is the leading cause of cancer and related deaths among women in Mozambique. The World Health Organization (WHO) recommends screening with human papillomavirus (HPV) testing, but it has not yet been implemented in many low- and middle-income countries (LMICs). We conducted a cervical cancer screening study in Mozambique using the careHPV test (QIAGEN, Germantown, MD, USA). This study aimed to describe our experience with HPV testing for cervical cancer screening in Maputo, Mozambique, including challenges and lessons learned.

\section{Methods}

We conducted a cervical cancer screening pilot demonstration project at the General Hospital of Mavalane in Maputo, Mozambique, from April 2018 to September 2019, using primary HPV testing with careHPV. We performed the direct observations and discussions with participating laboratory experts, technicians, nurses and physicians from Mozambique, Brazil and the United States.

\section{Results}

HPV testing was successfully implemented. The main challenges we experienced were inadequately equipped laboratory facilities, a lack of laboratory and clinical staff expertise, and difficulties procuring equipment, tests and laboratory supplies. These challenges were overcome by increasing the budget to better equip the laboratory, building relationships with procurement and importation specialists and training sessions for laboratory personnel and medical providers.

\section{Conclusions}

Our findings suggest that primary HPV testing is achievable in Mozambique. Several challenges were identified and are being addressed for a successful scale-up.

In Mozambique, cervical cancer is the leading cause of cancer and cancer-related deaths in women, with 5,325 new cases and 3,850 related deaths in 2020. ${ }^{1}$ The national screening program implemented in 2009 targets women aged 30-55 years, is opportunistic and uses visual inspection with acetic acid (VIA) followed by cryotherapy when indicated. ${ }^{2}$ The national coverage rate of screening has been estimated to be approximately $3 \% .^{2}$ In addition, the referral system and resources to treat women who require excisional treatment are very limited. Women with highgrade cervical abnormalities who are not eligible for cryotherapy often do not receive appropriate treatment. 
The World Health Organization (WHO) currently recommends screening women 30 years of age and older, prioritising women aged $30-49$ years. HPV testing is recommended where resources permit due to the increased sensitivity and high negative predictive value of HPV testing compared with cervical cytology and VIA. ${ }^{3}$ Based on WHO recommendations, we conducted a pilot study in Mozambique intending to perform HPV testing for cervical cancer screening, using the careHPV test (QIAGEN, Germantown, MD, USA $)^{4}$. The careHPV test is a user-friendly assay developed for limited-resource settings and shows a sensitivity greater than VIA and cervical cytology. ${ }^{3}$ In addition, careHPV recently received WHO prequalification. ${ }^{5}$ Advantages of the careHPV platform include 1) low cost compared to other HPV tests, 2) no need for sophisticated laboratory infrastructure, and 3) the relative ease of use. ${ }^{6}$ In addition, the only requirements are a flat, stable, non-flammable surface located out of direct sunlight and direct airflow, as well as three electrical outlets. ${ }^{7}$ The cost per test is approximately US $\$ 5$, provided specimens are run in batches of 90. This test cost is significantly lower than other existing HPV assays, which may cost US\$40-\$200 in the US. ${ }^{8}$ The purpose of this report is to describe our experience and lessons learned with the implementation of HPV testing at a public hospital in Maputo, Mozambique.

\section{SCENARIO}

The University of Texas MD Anderson Cancer Center, USA (MD Anderson) and the Universidade Eduardo Mondlane (UEM), Mozambique, conducted a cervical cancer screening pilot demonstration project of 898 women at General Hospital of Mavalane in Maputo, Mozambique from April 2018 to September 2019.4

Women aged 30 to 49 underwent pelvic exams; the cervical samples were collected by trained nurses. According to the manufacturer's instructions, the samples were tested for high-risk HPV using careHPV. Women who tested negative for HPV would return for routine screening per local guidelines. According to the WHO criteria, HPV-positive women underwent a second evaluation by visual assessment for treatment to determine whether they were eligible for ablative treatment with cryotherapy. If ineligible for cryotherapy, HPV-positive women were referred for colposcopy, biopsy, and excisional treatment with loop electrosurgical excision procedure or cold knife conisation as appropriate. Women with lesions suspicious for cancer underwent biopsy and were referred to the gynecologic oncology service for appropriate treatment.

\section{OUR EXPERIENCE, INCLUDING CHALLENGES AND LESSONS LEARNED}

\section{ETHICS BOARD APPROVALS}

In Mozambique, research projects and protocols require ethics committee approval, governed by local regulations. Unfortunately, our team did not allow sufficient time and resources to complete all necessary steps, which resulted in a delay in starting the project. Through this project, the collaborative team of researchers from Mozambique, Brazil, and the US understood the local ethics committee processes and regulations. This will help with future submissions. Furthermore, we learned that multiple approvals might be required from the hospital, university and/or ministry of health $(\mathrm{MOH})$. In our study, the protocol required both English and Portuguese versions and required approval from the US institution and the University and $\mathrm{MOH}$ in Mozambique.

\section{INFORMED CONSENT}

Several challenges were experienced in obtaining informed consent due to low literacy among some participants and the multiple languages and dialects spoken in Mozambique. Training and informational sessions were held between the Mozambican, Brazilian and US research staff to meet all requirements by the ethics boards and local customs. In addition, mock consent interviews were conducted with the nursing and research staff to practice the process and learn to answer complex questions from the subject.

\section{SUPPORT FROM HOSPITAL LEADERSHIP}

The support from the institution and hospital director was essential to addressing the challenges related to local infrastructure and appropriate allocation of local staffs time to perform the project activities. Security of equipment, samples, and data were essential considerations. Equipment, computers, and records needed to be stored in an area that could be locked. At the General Hospital of Mavalane, we received strong support from the hospital director, enabling the project to move forward safely, with a high level of support in infrastructure, human and facility resources.

\section{MENTORING AND REMOTE SUPPORT}

The Brazilian and US teams helped support the study from their home institutions. Unfortunately, the hospital internet in Mozambique was not reliable. This was overcome by purchasing devices and data packages so that the local research and clinical staff could participate in weekly virtual meetings to continue training and manage and discuss any difficulties, including issues with data entry.

\section{DATA COLLECTION AND DATA ENTRY IN RESEARCH ELECTRONIC DATA CAPTURE (REDCAP)}

This was the first cervical cancer screening research study at the General Hospital of Mavalane. The local researchers and clinical providers did not have prior experience conducting a research study but were enthusiastic about learning and participating in the project. The team was trained to perform data collection and entry into a REDCap electronic data capture tool hosted at The University of Texas MD Anderson Cancer Center. Brazilian and US collaborators performed the training and weekly quality control reviews on the REDCap database to help identify and correct data entry errors. Study data were collected and managed using 
REDCap reports, Data Quality, and Data Resolution Workflow applications to control and manage data accuracy. ${ }^{9}$ The research staff in Mozambique became very skilled using REDCap, and data entry became increasingly more accurate throughout the study.

\section{LABORATORY INFRASTRUCTURE}

Prior to selecting the testing platform and the start of the project, a site visit was conducted to select the appropriate space, considering the physical structure, power supply, availability of water, air conditioning, and bench and counter space. We identified a suitable room close to the testing site, but upgrades to the power supply, an air-conditioning unit, shelf storage, refrigeration, and freezer storage were required. Re-budgeting was necessary to implement the upgrades to the laboratory necessary to perform the testing.

To ensure a controlled laboratory environment, one 2-8 ${ }^{\circ} \mathrm{C}$ refrigerator for samples and test kits storage, one -20 ${ }^{\circ} \mathrm{C}$ freezer for storage of samples, and one air conditioning unit were installed and underwent daily temperature verification. Unfortunately, the electrical power in the laboratory was unstable. As a result, we experienced two power failures while running tests, invalidating the 90 patient samples tested in each kit and requiring additional time and supplies to repeat the test. An uninterrupted power supply (UPS) was connected to the careHPV system to prevent further failures.

\section{SUPPLIES}

Most of the required laboratory supplies (e.g. micropipettes, repeating pipettes, and pipette tips) were not available for purchase in Mozambique and had to be imported. The importation process was complicated and required importation and clearance through customs. The associated fees, including shipping costs, were very high at approximately US $\$ 1,300$. Deliveries of supplies were frequently delayed by two months or more. We partnered with the Clinton Health Access Initiative (CHAI) to assist with procurement and importation challenges to overcome these challenges.

\section{TRAINING OF LABORATORY AND CLINICAL PERSONNEL}

The laboratory technicians at Mavalane Hospital had no prior experience with molecular testing and were trained to perform the careHPV test by laboratory experts from Brazil and Qiagen. Technical support personnel from Qiagen provided training for one laboratory technician using the careHPV test training panel. Two additional technicians underwent one to four one-week training sessions conducted by laboratory specialists from Brazil using the cervical specimens collected for the study.

There was variability in the learning curve between the three laboratory technicians. The technicians received training and mentoring from Brazilian laboratory experts. For those laboratory technicians that experienced difficul- ties in pipetting techniques, multiple practice sessions of pipetting water in a 96 well plate were held.

Hands-on training sessions were also held for the clinical providers (nurses and doctors) to learn how to collect cervical samples for HPV testing and how to perform VIA, cryotherapy, colposcopy, and LEEP. Furthermore, regular didactic sessions were held for both the laboratory and clinical staff and covered topics such as an overview of the different HPV tests available and different aspects of cervical cancer screening and treatment.

\section{RECOMMENDATIONS}

Based on our experiences, several aspects should be considered before implementing HPV testing. First, in a program comprising both local and external participants, the local leadership and staff must be committed to ensuring the project needs are met and have sufficient support to meet the goals.

It is crucial to understand the local regulations and procurement procedures during the study design process to avoid delays. Moreover, it is essential to assess the lab to ensure safe and adequate infrastructure before delivering any HPV testing equipment for a cervical cancer screening program. ${ }^{6}$ The budget should include funds to cover high shipment costs and importation fees. In addition, budgets should include funding to implement and support required site improvements, especially in the laboratory infrastructure. Funds are also needed to acquire individual protection equipment (IPEs), computers and internet access.

It is vital to assess the laboratory technicians experience and abilities prior to project implementation. Appropriate training sessions and ongoing mentoring and support can then be provided. Regular communication is needed, particularly if there is limited local expertise in the country and external support is needed. In our project, weekly meetings were held by Zoom between Mozambique, Brazil, and the US teams. Furthermore, regular training sessions may be required to avoid loss of skills, particularly when project activities are paused.

\section{FUNDING}

The authors would like to thank Qiagen for providing the careHPV system for the study. This study was supported in part by the National Institutes of Health through MD Anderson's Cancer Center Support Grant P30CA016672; The Anadarko Petroleum Corporation; The Prevent Cancer Foundation; The Dunaway Family Fund; The Joe Family Fund; and The Giles-O’Malley Foundation.

\section{AUTHORSHIP CONTRIBUTIONS}

EB, MPS, JHTGF, KMS, RRRK and CL were involved in the conceptualisation, project administration and supervision. $\mathrm{CMO}, \mathrm{EB}, \mathrm{KMS}$ and MPS were involved in writing the original draft, formal analysis and investigation. NP, EM, AM, MCV, RAMJ, KK, CS, VA and JC were involved in the 
methodology and investigation. VA were also involved in data curation and validation. All authors reviewed, edited, and approved the final manuscript.

\section{COMPETING INTERESTS}

The authors completed the Unified Competing Interest form at http://www.icmje.org/disclosure-of-interest/ (available upon request from the corresponding author), and declare no conflicts of interest.

\section{CORRESPONDENCE TO:}

Cristina Mendes de Oliveira, $\mathrm{PhD}$.

Av. Juruá, 548 - Dasa laboratories, Barueri, Brazil. Phone: +551126302770.

cristina.oliveira1@gmail.com

Submitted: August 18, 2021 GMT, Accepted: January 11, 2022

GMT 


\section{REFERENCES}

1. Bray F, Ferlay J, Soerjomataram I, Siegel RL, Torre LA, Jemal A. Global cancer statistics 2018: GLOBOCAN estimates of incidence and mortality worldwide for 36 cancers in 185 countries: Global Cancer Statistics 2018. CA: A Cancer Journal for Clinicians. Published online September 12, 2018. do $\mathrm{i}: 10.3322 /$ caac. 21492

2. Brandão M, Tulsidás S, Damasceno A, Silva-Matos C, Carrilho C, Lunet N. Cervical cancer screening uptake in women aged between 15 and 64 years in Mozambique: European Journal of Cancer Prevention. 2019;28(4):338-343. doi:10.1097/CEJ.0000000000000 $\underline{459}$

3. Mustafa RA, Santesso N, Khatib R, et al. Systematic reviews and meta-analyses of the accuracy of HPV tests, visual inspection with acetic acid, cytology, and colposcopy. International Journal of Gynecology \& Obstetrics. 2016;132(3):259-265. doi:10.1016/j.ijgo.20 15.07.024

4. Salcedo MP, Oliveira C, Andrade V, et al. The Capulana study: a prospective evaluation of cervical cancer screening using human papillomavirus testing in Mozambique. International Journal of Gynecologic Cancer. 2020;30(9):1292-1297. doi:10.1136/ijgc-202 $\underline{0-001643}$
5. WHO. WHO Prequalification of In Vitro

Diagnostics. Published 2018. https://www.who.int/dia gnostics laboratory/evaluations/pq-list/180713_pqp r_pqdx_0085_028_00_carehpv_with_labelling.pdf?ua =1

6. Jeronimo J, Bansil P, Lim J, et al. ATL. International Journal of Gynecologic Cancer. 2014;24(3):576-585. do i:10.1097/IGC.0000000000000084

7. Qiagen. CareHPV Test System User Manual. Published online December 2015.

8. Centers for Medicare \& Medicaid Services. Clinical Laboratory Fee. Accessed February 15, 2020. http s://www.cms.gov/medicaremedicare-fee-service-pay mentclinicallabfeeschedclinical-laboratory-fee-sched ule-files/20clabq2

9. Harris PA, Taylor R, Thielke R, Payne J, Gonzalez N, Conde JG. Research electronic data capture (REDCap)--a metadata-driven methodology and workflow process for providing translational research informatics support. J Biomed Inform. 2009;42(2):377-381. doi:10.1016/i.jbi.2008.08.010 